\title{
Development of electrochemical sensor based on silica/gold nanoparticles modified electrode for detection of arsenite
}

\begin{abstract}
Arsenic is an extremely poison element in earth crust and its contamination in environment is a global hazard. In this study, an efficient electrochemical detection of arsenite [As(III)] has been developed using linear sweep anodic stripping voltammetry (LSASV), based on adsorption of arsenic on the surface of screen printed carbon electrode modified silica/gold nanoparticles (SiNPs/AuNPs/SPCE). The surface property of modified electrode was characterized by field emission scanning electron microscopy (FESEM), transmission electron microscopy (TEM), energy dispersive X-ray spectroscopy (EDX) and fourier transform infrared spectroscopy (FTIR). The morphology studies using FESEM showed that the distribution of SiNPs/AuNPs composite is not homogenous therefore resulting in some areas with aggregation on the working electrode surface. Several optimum voltammetric parameters were established such as supporting electrolyte, $1 \mathrm{M} \mathrm{HCl}$; deposition potential, $-0.4 \mathrm{~V}$ and deposition time, $300 \mathrm{~s}$. Under optimum condition, a linear correlation was obtained in the range of $10-100 \mathrm{ppb}$ with limit of detection $5.6 \mathrm{ppb}$. A variety of common coexistence ions such as $\mathrm{Pb} 2+, \mathrm{Ni} 2+, \mathrm{Zn} \mathrm{2+}, \mathrm{Hg} 2+$ and $\mathrm{Cu} 2+$ in water samples showed no interferences in arsenite detection. The proposed method showed high sensitivity and good reproducibility with a relative standard deviation of $4.52 \%$, providing potential application of arsenite detection in environment.
\end{abstract}

Keyword: Arsenic; Gold nanoparticles; Screen printed carbon electrode; Silica nanoparticles 